Background: Chronic intestinal pseudo-obstruction can be a rare complication of systemic lupus erythematosus. It is often late to be identified in contrast to other commoner organs involvement in systemic lupus erythematosus such as nervous system, joint and kidney.

Objectives: To report a case of uncommon gastrointestinal complication of lupus, with associated hydronephrosis at the ureter, with treatment delay.

Methods: We report a case of chronic intestinal pseudo-obstruction.

Results: A 34 year old, with ten-year history of lupus nephritis, presented with recurrent abdominal pain and diarrhoea past nine months. The patient had just been diagnosed as end-stage renal failure a year ago, on regular haemodialysis. For the past ten years, the patient was not able to tolerate immunosuppressant due to the multiple episodes of infections, including shin carbuncle, herpes zoster, breast abscess and catheter-related candida/bacterial infections. However, the patient did not have any other major organs manifestations of lupus for the past ten years. The patient was apyretic. Multiple stool cultures were negative including Clostridium difficile. The abdominal radiography showed dilated small bowel with diffuse thickening of large and small bowels on computed tomography. Bilateral uretero-hydronephrosis was also noted without any evidence of obstructive uropathy on imaging. Ileocolic resection was done for presumed intestinal obstruction and the ileocolic biopsy did not reveal any granuloma, malignancy or vasculitis except for non-specific inflammation of cecum. Cytomegalovirus inclusion body was absent as well. Tuberculosis culture was negative. Oesophagoduodenoscopy and colonoscopy were offered in view of persistent unexplained loose stool and abdominal pain. But unremarkable findings were noted from multiple biopsy specimens of the small and large bowels. Second relook of the initial hemicolectomy specimen with special actin immunostain on the smooth muscle revealed degenerative changes of the muscularis propria. These were evidenced by cytoplasmic vacuolation, atrophy and pyknotic nucleus of the smooth muscle cells with surrounding oedema. Smooth muscle dysmotility could be the underlying pathology of this patient presentation. The patient responded well to intravenous immunoglobulin followed by azathioprine in addition to prednisolone and prokinetic agent.

Conclusion: Prompt recognition is pivotal in this case could have prevented the unnecessary surgical intervention earlier. It is potentially reversible. Long term prognosis of this rare entity is, however, varying.

Disclosure of Interests: None declared

DOI: 10.1136/annrheumdis-2021-eular.2854

\section{AB0857 CERTOLIZUMAB PEGOL RELOADING SUCCESSFULLY CONTROLS INFLAMMATORY ARTHRITIS FLARES DURING PREGNANCY - A CASE REPORT}

V. Pavlova ${ }^{1} .{ }^{1}$ McMaster University, Department of Medicine, Hamilton, Canada

Background: Approximately $50 \%$ of patients with inflammatory arthritis (IA) experience worsening of disease activity during pregnancy. ${ }^{1,2}$ Given the evidence that increased disease activity immediately before or during pregnancy is associated with adverse pregnancy outcomes, it is imperative to maintain tighter disease control during this period. ${ }^{3}$ Treatment options are limited to steroids or disease-modifying anti-rheumatic drugs (DMARDs) compatible with pregnancy. Certolizumab pegol (CZP) is a humanized TNF-alpha inhibitor with no to minimal transplacental transfer, making it a suitable option during pregnancy. ${ }^{4}$ In patients who relapse while on treatment with a biologic, consideration can be given to optimizing the dose of the biologic. Studies in Crohn's disease demonstrated that increasing the CZP dose from $400 \mathrm{mg}$ Q4W to Q2W is a viable strategy for recapturing efficacy. ${ }^{5}$ Similar data is not available for the rheumatology population or for pregnancy.

Objectives: To describe the successful control of juvenile inflammatory arthritis (JIA) flares using a reloading strategy with CZP in a pregnant patient with prior failure of multiple conventional systemic (cs)DMARDs and biologics.

Methods: This is a case report of a 31-year old female with JIA who presented with a disease flare in the $1^{\text {st }}$ trimester of her $2^{\text {nd }}$ pregnancy. The patient was treated with multiple csDMARDs and biologics since age 6. She experienced significant gastrointestinal side effects to subcutaneous methotrexate and leflunomide and inadequate response to hydroxychloroquine and sulfasalazine. Subsequently, she initiated and maintained treatment with etanercept for 14 years until she developed secondary loss of efficacy. She had primary non-response to golimumab and abatacept and experienced a severe allergic reaction to infliximab. Since 2017, she has been treated with CZP $200 \mathrm{mg}$ Q2W with good control of disease activity.

Prior to conception, the patient had low disease activity (Table 1). At 11 weeks gestation, the patient noted worsening of disease activity, with pain at 4/10, fatigue at $7 / 10$, morning stiffness for 60 minutes, swollen joint count (SJC)
4/28, and tender joint count (TJC) 19/28. The patient global assessment of disease activity (PtGA) remained at 4/10 while physician global assessment of disease activity (PhGA) and health assessment questionnaire disability index (HAQ-DI) increased to $6 / 10$ and 1.625 , respectively (Table 1). She reported that symptoms worsen one week after CZP injection with more joint pain and swelling in hands, wrists, and feet. The patient declined the addition of hydroxychloroquine, sulfasalazine, and steroids due to previous tolerability, lack of efficacy, and concerns about safety during pregnancy. The patient received 3 loading doses of CZP $400 \mathrm{mg}$ Q2W. She then continued treatment with CZP $200 \mathrm{mg}$ Q2W.

Table 1. Disease activity prior to conception and during pregnancy before and after reloading CZP.

\begin{tabular}{lccc}
\hline Characteristic & Prior to conception & 11 weeks gestation & $\begin{array}{c}19 \text { weeks gestation } \\
\text { after reloading CZP }\end{array}$ \\
\hline SJC (28 joints) & 2 & 4 & 2 \\
TJC (28 joints) & NA & 19 & NA \\
PhGA* & 4 & 6 & 2 \\
PtGA* $^{*}$ & 4 & 4 & 4 \\
HAQ-DI & 1.5 & 1.625 & 1.5 \\
Pain* & 4 & 4 & 2 \\
Fatigue* $^{*}$ & 7 & 7 & 5 \\
Morning stiffness (min) & 60 & 60 & $20-30$ \\
\hline
\end{tabular}

${ }^{\star}$ Assessed on a 10-point scale.

Results: On follow-up at 19 weeks gestation the patient reported $80 \%$ improvement in joint pain and swelling and reduction in morning stiffness (Table 1). Pain and fatigue were rated as $2 / 10$ and $5 / 10$, respectively. PtGA score was $4 / 10$, PhGA score was 2/10 and HAQ-DI score was 1.5 (Table 1).

Conclusion: Reloading CZP to control increased IA disease activity during pregnancy may be a viable treatment option. To our knowledge, this is the first report of dose optimization in IA and in pregnancy in a patient treated with CZP.

\section{REFERENCES:}

[1] van den Brandt S, et al. Arthritis Res Ther 2017;19(1):64.

[2] Jethwa, $\mathrm{H}$ et al, Arthritis Rheumatol. 2016;68 (suppl 10).

[3] de Man Y, et al. Curr Opin Rheumatol. 2014;26:329-333.

[4] Mariette X, et al. Ann Rheum Dis. 2018 Feb;77(2):228-233.

[5] Sandborn WJ, et al. American College of Gastroenterology Annual Scientific Meeting. 2009; Abstract 699

Disclosure of Interests: Viktoria Pavlova Speakers bureau: Amgen, Abbvie, BMS, Jenssen, Lilly, Merk, Novartis, Roche, UCB, Pfizer, Consultant of: Amgen, Abbvie, BMS, Jenssen, Lilly, Merk, Novartis, Roche, UCB, Pfizer, Grant/research support from: UCB

DOI: 10.1136/annrheumdis-2021-eular.3410

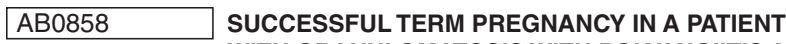 WITH GRANULOMATOSIS WITH POLYANGIITIS AFTER UNDERGOING RITUXIMAB THERAPY - CASE REPORT}

O. Sirenko ${ }^{1}$, O. Kuryata ${ }^{1}$, T. Lysunets ${ }^{2}$, A. Legkobyt ${ }^{2}$, H. Mostova ${ }^{3}$.

${ }^{1}$ Dnipropetrovsk Medical Academy, Internal Medicine 2, Dnipro, Ukraine; ${ }^{2}$ Dnipropetrovsk Regional Clinical Hospital After Mechnicov, Rheumatology, Dnipro, Ukraine; ${ }^{3}$ Dnipropetrovsk Regional Clinical Hospital After Mechnicov, Obstetrics and Gynecology, Dnipro, Ukraine

Background: Granulomatosis with polyangiitis (GWP) is a relatively rare disease with the lack of experience in the management of pregnancy currently. Potentially negative factors of pregnancy prognosis are both disease-related and teratogenic effects of specific vasculitis therapy [1].

Objectives: To describe a clinical case of Successful term pregnancy in a patient with Granulomatosis with polyangiitis after undergoing rituximab therapy. Methods: 19 years-old woman was admitted to the rheumatology department of Clinical Regional Hospital After Mechnicov in 2013 with the debut of Granulomatosis with polyangiitis. Her disease course included recurrent rhinitis and sinusitis, Granulomatosis of the eye orbits. Positive ANCA titers were present in the disease onset. In the preceding 7 years she had been treated with corticosteroids, cyclophospan (discontinuated in 2017 because of lack of efficiency), than azathioprine. Aseptic necrosis of the both femoral head was estimated on fifth disease year. In 2018 azathioprine therapy had been discontinued owing to the disease progression and biological agents were prescribed - rituximab with positive effect. The disease remission was achived by rituximab therapy, the patient was warned about the need for contraception. The last dose of rituximab was introduced in February 2020. However, in March 2020, the patient reported pregnancy and therapy was discontinued. At the onset of pregnancy she was 
treated with $6 \mathrm{mg}$ oral methylprednisolone. Daily and this dose was stable till all pregnancy period. Fetal growth assessment, congenital abnormalities screening test, and laboratory tests for gestational diabetes and preeclampsia were unremarkable during all gestational trimesters. The ANCA titers remained negative and renal function was normal and there was no flare during all pregnancy period.

Results: At 38 weeks' gestation a spontataneous labor started without induction. A 3270-g healthy boy delivered with APGAR score of 9 at 1 minute and 5 minutes. Postpartum the disease remained in remission, and the patient was maintained on corticosteroids. The infant was healthy, with normal development. Conclusion: Thus, the use of biological agents therapy in patients with GWP shows possible ways to safe reproductive potential with disease remission achievement.

\section{REFERENCES:}

[1] Daher A, Sauvetre G, Girszyn N, Verspyck E, Levesque H, Le Besnerais M. Granulomatosis with polyangiitis and pregnancy: A case report and review of the literature. Obstetric Medicine. 2020;13(2):76-82. doi:10.1177/17534 $95 \times 18822581$

Disclosure of Interests: None declared

DOI: 10.1136/annrheumdis-2021-eular.4016

\section{HPR Measuring health (development and measure- ment properties of PROs, tests, devices)}

\section{AB0859-HPR NETWORK OF SUPPORT AND DEPRESSION IN RHEUMATIC DISEASES}

L. Cano Garcia ${ }^{1}$, S. Manrique Arija ${ }^{1}$, F. Godoy-Navarrete ${ }^{1}$, F. G. JiménezNúñez ${ }^{1}$, R. Redondo ${ }^{1}$, N. Mena-Vázquez ${ }^{1}{ }^{1}$ Hospital Regional Universitario de Málaga, Rheumatology, Málaga, Spain

Objectives: To describe the impact that depression has on the personal support network of patients with rheumatic diseases.

Methods: Design. Cross-sectional observational study of a series of patients with spondyloarthritis $(\mathrm{SpA})$, rheumatoid arthritis (RA) and systemic lupus erythematosus (SLE) selected by consecutive sampling of the Rheumatology consultations. Selection criteria: age $\geq 18$ years with $A D$ (ASAS criteria), RA (EULAR / ACR 2010 criteria) and SLE (ACR-EULAR criteria) capable of understanding and willing to take the questionnaires. Protocol: The reference rheumatologist offered to participate in the study to all the patients who attended the consultation between October and December 2019 and met the selection criteria. After their approval and signing the informed consent, they went to the nursing consultation to carry out a battery of questionnaires from the PROMIS platform and collect clinical data. The infirmary was in charge of explaining the questionnaires to the patients. Variables: the main endpoint variable was depression evaluated by PROMIS and the secondary endpoint variables were questionnaires that evaluate the support network in various settings: company, emotional support, instrumental support, support through information. Likewise, data on their disease, comorbidities were collected using Charlson and socio-occupational profile. Statistical analysis: Descriptive analysis, R-Pearson correlation and multivariate linear regression analysis (VD: PROMIS depression).

Results: 151 patients participated: 50 with RA (90\% women, mean age $55.12 \pm$ 13.64 years), 51 with $A D$ ( $51 \%$ women, $52.59 \pm 12.15$ years) and 50 patients with SLE (96\% women, mean age $47.14 \pm 11.3$ years). The baseline characteristics and comorbidities of the included. The mean (SD) of PROMIS depression in all patients was $16.4(8.3)$ and a total of 65 patients (43\%) had depression according to the Charlson index. Patients with Charlson depression had a higher mean (SD) of PROMIS depression than those without Charlson depression (24.5 [6.2] vs 10.3 [2.5], $p<0.001$ ). An inverse correlation was observed between PROMIS depression with the rest of PROMIS questionnaires: company $(r=-0.369, p=$ $<0.001)$, emotional support $(r=-0.533, p=<0.001)$, information support $(r=$ $-0.577, p=<0.001)$ and instrumental support $(r=-0.362, p=<0.001)$. Likewise, it was observed that patients without depression by Charlson compared with patients with depression had higher mean values $(\mathrm{SD})$ in all questionnaires of the support network compared to those without depression by Charlson: company (17, 8 (2.9) vs 14.7 (4.7), p <0.001), emotional support (37.2 (5.2) vs 29.0 (9.5), $p<0.001$ ) and instrumental support (35.7 (7.2) vs 29.4 (8.8), $p<0.001)$. Finally, in the multivariate analysis, the variables that were independently associated with depression by PROMIS were information support $(B=-0.390 ; p<0.001)$ and emotional support $(B=-0.239 ; p=0.027)$. $(R 2=0.340)$

Conclusion: Depression in rheumatic diseases negatively influences the support network and the perception of company of these patients. Treating depression is important for social functioning and social relationships in these patients and for any group of chronic patients.

Disclosure of Interests: None declared

DOI: 10.1136/annrheumdis-2021-eular.1167

\section{AB0860-HPR IS THERE A RELATIONSHIP BETWEEN PHYSICAL ACTIVITY, SLEEP QUALITY, ANXIETY AND DEPRESSION IN PATIENTS WITH FAMILIAL MEDITERRANEAN FEVER?}

T. Civi Karaaslan ${ }^{1}$, S. Ugurlu' ${ }^{2}$ E. Tarakci ${ }^{1} .{ }^{1}$ Faculty of Health Sciences, Physiotherapy and Rehabilitation, Istanbul, Turkey; ${ }^{2}$ Cerrahpasa Faculty of Medicine, Department of Internal Medicine, Division of Rheumatology, Istanbul, Turkey

Background: Maintenance of regular physical activity is associated with bette physical and mental health (1). In addition, sleep disturbances and mood disorders are common in chronic inflammatory diseases (2).

Objectives: The aim of this study was to determine the relationship between physical activity, sleep quality, anxiety and depression in patients with FMF. Methods: A total of 56 patients ( 30 female, 26 male) with Familial Mediterranean Fever (FMF) were enrolled in the study. They were diagnosed with FMF based on the Livneh diagnostic criteria (3). International Physical Activity Questionnaire - Short Form (IPAQ) was used to evaluate health-related physical activity. Patient-reported sleep quality was evaluated with the Pittsburgh Sleep Quality Index (PSQI). Hospital Anxiety and Depression Scale (HADS) was used to evaluate anxiety and depression in FMF patients. Patients who were 20-55 years old was included in the study.

Results: The mean age was $32.23 \pm 9.87$ years. The mean disease duration of the patients was $12.24 \pm 7.61$ years. The education level of $51.8 \%(n=29)$ of the participants was undergraduate and above. The rate of those who did not work was $32.1 \%(n=18)$ and $48.2 \%(n=27)$ of the participants were married. The mean of scores of IPAQ was $2333.99 \pm 2640.29$, the mean of PSQI was $6.14 \pm 3.66$, the mean of HADS-Anxiety was $7.89 \pm 5.03$ and HADS-Depression was $7.01 \pm 4.44$ The patients were classified according to physical activity categories as low $(25.0 \%, n=14)$, moderate $(41,1 \% n=23)$ and high $(33.9 \%, n=19)$. Physical activity categories were not found associated with PSQI $(p=0.437)$, HADS-Anxiety $(p=0.363)$ and HADS-Depression $(p=0.861)$. The relationships of scores of IPAQ, PSQI and HADS were demonstrated Table 1.

Conclusion: This study confirmed that patients with FMF suffer from sleep disturbances, anxiety and depression. In addition, sleep disturbances, anxiety and depression were not associated with physical activity category.

\section{REFERENCES:}

[1] Sokka, Tuulikki, et al. Physical inactivity in patients with rheumatoid arthritis: data from twenty-one countries in a cross-sectional, international study. Arthritis Care \& Research: Official Journal of the American College of Rheumatology, 2008, 59.1: 42-50.

[2] Kucuksahin, Orhan, et al. Incidence of sleep disturbances in patients with familial Mediterranean fever and the relation of sleep quality with disease activity. International journal of rheumatic diseases, 2018, 21.10: 1849-1856.

[3] Bashardoust, Bahman. Familial Mediterranean fever; diagnosis, treatment, and complications. Journal of nephropharmacology, 2015, 4.1: 5.

Table 1. The correlations of IPAQ, PSQI and HADS scores.

\begin{tabular}{lcccc}
\hline & & HADS-Depression & HADS-Anxiety & PSQI \\
\hline IPAQ & $\mathrm{r}$ & -0.091 & -0.142 & -0.002 \\
& $\mathrm{p}$ & 0.505 & 0.295 & 0.990 \\
PSQI & $\mathrm{r}$ & 0.689 & 0.615 & \\
& $\mathrm{p}$ & 0.001 & 0.001 & \\
HADS-Anxiety & $\mathrm{r}$ & 0.681 & & \\
& $\mathrm{p}$ & 0.001 & & \\
& & &
\end{tabular}

-Pearson Correlation

Disclosure of Interests: None declared DOI: 10.1136/annrheumdis-2021-eular.1290 